\title{
Studying the Coefficient Curvelet for Aerial Image Segmentation
}

\author{
Nagham A. Sultan \\ naghamajeel@uomosul.edu.iq \\ College of Computer Science and Mathematics \\ Khalil I. Al-Saif \\ khalil_alsaif@hotmail.com \\ University of Mosul, Mosul, Iraq
}

Received on: 30/10/2019

Accepted on: 09/01/2020

\begin{abstract}
Currently, there are many approaches for image transformations that are developed to cover the new technology in the huge amount of data treatment. In this paper, a study on Curvelet transformation coefficients was performed based on the aerial image to apply segmentation. This paper applies a lot of modifications on cut off frequencies on the filters, which is used to decompose the image on curvelet transformation. Two approaches are proposed and tested to look for the best segmentation result; the first one is based on designing filters manually, while the second evaluates the filter coefficients depending on the selected shape of the filters. The first technique gives acceptable segmentation and the second reaches the optimal result. One of the most important results is that the cut of frequency has a high effect on the segmentation; in addition, choosing filter parameters depended on the coefficients dimension of the curvelet transformation. Finally, the results show that the first approach underperformed the second one.
\end{abstract}

Keywords: Curvelet Transform, Directional Filter Bank, Pyramidal Directional Filter Banks, Curvelet Coefficient, Aerial Image.

$$
\begin{aligned}
& \text { دراسة معاملات كيرفليت لتقطيع الصور الجوية } \\
& \text { خليل إبراهيم السيف } \\
& \text { كلية علوم الحاسوب والرياضيات } \\
& \text { جامعة الموصل، الموصل، العراق }
\end{aligned}
$$

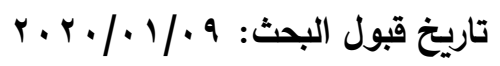

$$
\begin{aligned}
& \text { تاريخ استلام البحث: • ب/ • 19/1 r } \\
& \text { نغم عجيل سلطان }
\end{aligned}
$$


الكلمات المفتاحية: تحويلات كيرفليت، المرشح الاتجاهي، المرشح الهرمي الاتجاهي، معاملات كيرفليت، الصور الجوية.

\section{Introduction}

The wavelet transformation and Gabor filters limit by new techniques. One of these techniques can be applied to the curvelet transformation. Though, image processing adopts widely wavelet transformation in various branches and aspects. Wavelet transform fail to represent whole edge and curves. Also, it is not appropriate to detect line singularities. Gabor filter is better than wavelet in image retrieve and also better in texture representation, therefor it is multiple orientation approach. Also Gabor filter cannot represent images effectively due to the loss of spectral information. According to the aforementioned description, curvelet has been developed to achieve a complete coverage capture and more orientation information and spectral domain [4], and The curvelet transform which represents edges better than wavelets because edges play a fundamental role in image understanding, one good way to enhance spatial resolution is to enhance the edges. Curvelet-based image fusion method provides richer information in the spatial and spectral domains simultaneously [11]. The first generation of discrete curvelet transform was given by Starck et al. "To cover specialized for image processing applications. However, in the past few years, curvelets have been redesigned to make them easy to use and understand. Two dimensions discrete curvelet transforms was presented by Cand es as second-generation curvelets. These new transforms are numerically tight frames, and the resulting curvelets are faithful analogs of their continuous Counterparts" [6]. The main aim of the paper is to establish a link between curvelets and filter bank parameters, then, study a new relation between all the parameters of the filter bank structure (named pyramidal directional filter banks (PDFB)), which achieves in images decomposition based on Curvelet-like image decomposition.

This paper is organized as follows: the next section highlights on the related works belongs to our work, while section 3 presents the principles of curevelet transform .section four explains the proposed algorithm in two manner with practical implementation, finally conclusion and future work are listed in section 5.

\section{Related Work}

A. Djimeli, D. Tchiotsop et.al in 2013 enhanced the edge model based on Curvelet coefficients analysis. The new method illustrated that the method brings out details on edges when the decomposition scale increases [3].

Kunal Pandey and Shekhar Suralkar in 2013 proposed a characteristic of the Fast Discrete Curvelet Transform hybrided by an image fusion algorithm based on Discrete Wavelet Transform and the Fast Discrete Curvelet Transform has been analyzing [5].

Tirveedi Prasad and Ch.Pullarao in 2014 presented an approach to design and enhance image resolution using zero padding and curvelet transform. The proposed algorithm used curvelet transform for multilevel image decomposition, then adding picture with high frequency then merge images using inverse curvelet transform (IFDCT). The algorithm was tested on a number of standard images and gives result vision and Peak signal-to-noise ratio (PSNR) better than traditional methods for image enhancement [10].

Raju C. et.al in 2016 presented a method for denoising of remotely sensed images via curvelet transform and its relative assessment. In this paper, the denoising of 
remotely sensed images based on Fast Discrete Curvelet Transform (FDCT) has been proposed. "With its optimal image reconstruction capabilities, the curvelet outperforms the wavelet technique in terms of both visual quality and Peak Signal to Noise Ratio (PSNR). This paper focuses on the analysis of denoising the Linear Imaging Self Scanning Sensor III (LISS III) images, Advanced Very High Resolution Radiometer (AVHRR) images from National Oceanic and Atmospheric Administration 19 (NOAA 19), METOP satellites for the Tirupati region, Andhra Pradesh, India. Numerical illustrations demonstrated that this method is highly effective for denoising the satellite images" [12].

\section{Curvelet Transform}

Candes and Donoho presented an idea for the first curvelet transform in 1999 [13]. It was very slow, and its method was developed by not using ridgetettransform, thus becoming fast and called fast discrete curvelet transform, the following steps show how can applying discrete curvelet transform[1]:

1- Origin image split up in three sub bands.

2- Tiling is performed on sub bands $\Delta 1 \& \Delta 2$.

3- Discrete Ridge let transform is performed on tile.

The initial method of curvelet transform implements the concept of discrete ridgelet transform. Since its creation in 1999, Ridgelet transform based curvelet transform has been successfully used as an effective tool in image decomposition, image de-convolution, texture classification, image de-noising, contrast enhancement, and astronomical imaging, etc. However, ridgelet-based curvelet transformation is not efficient as it uses complex ridgelet transform [4]. In 2004 Candes and Donoho reconstructed second generation curvelet transform. Then, in 2005, "a fast discrete algorithm was added to the second generation transform by Candes et al. This curvelet transform is not only a kind of multiscale geometric transform with frame element indexed by scale, location and strong direction parameters in the frequency domain, but also easier manipulation, much simpler structure, and less redundancy than first generation. There are two digital implementations of the second generation curvelet transform: The first one is Fast Discrete Curvelet Transform (FDCT) via Unequally Spaced Fast Fourier Transform (USFFT), and the FDCT via wrapping. Both the implementations take as inputting a Cartesian array to obtain an output of curvelet coefficients" [13].

The Wrapping based curvelet transform is faster in computation time and more robust than ridgelet transform and USFFT based curvelet transform [4]. Curvelet transform has been presented as an attractive technique for various applications. The Curvelet Transform is based on decomposing the image into different scales, then partitioning into squares whose sizes based on the corresponding scale, The curvelet transform is suited for the images that contains edges, therefore, it is a good for edge enhancement. Curvelet coefficients can by modified in order to enhance edges in an image by $\mathrm{y}(\mathrm{x})$ (Velde function). This function aims to faint edges [7]. Two"dimensional Curvelets waveforms provide a new building for multi-scale analysis. In space domain curvelet at scale $\mathrm{j}$ is an oriented needle whose effective support is a $2^{-\mathrm{j}}$ by $2^{-\mathrm{j} / 2}$ rectangle and thus submits parabolic scaling relation width $=$ length $^{2}$ while, in frequency domain curvelet at scale $\mathrm{j}$ is a wedge whose frequency support is again inside a rectangle, but of size $2^{\mathrm{j}}$ by $2^{\mathrm{j} / 2}$. Dissimilar wavelets, Curvelets are localized not only in position "the spatial domain" and scale "the frequency domain", but also in orientation" [6]. 
Harmonic scales curvelet transform is a directional and local decomposition of data (image) and aims to discover the contribution from each point of data in the $t-x$ domain to isolate directional windows in the $\mathrm{f}-\mathrm{k}$ domain. The width and length of the support of a curvelet satisfy the parabolic scaling relation length $=$ width $^{2}$ in the wave number domain, because of this anisotropic scaling the curvelets become line-like in the limit of infinite frequency. Note that parabolic scaling relation is length $=$ width $^{2}$ when in the spatial domain [14].

\subsection{Curvelet Properties \& Application}

The curvelet properties can be summarized as follows [2]:

- Curvelet transform is based on a new kind of pyramid filter structure.

- Frame elements of the Curvelet exhibit new scaling laws.

- Curvelet provides an efficient representation of images with edges that can be provided by Curvelet.

Recently, curvelet has become more popular in different research areas. Particularly, the development of FDCT in 2006 had a profound impact on a variety of applications such as image fusion, texture analysis, contrast enhancement, Morphological Component Analysis, image restoration, image enhancement, image compression, compressed sensing, image denoising, seismic data exploration, image deconvolution, watermarking, astronomical image representation, palmprint recognition, face recognition, finger-vein pattern recognition, optical character recognition [15].

\subsection{Curvelets Filter Banks}

The Pyramidal Directional Filter Banks (PDFB) uses appropriate combination of the directional filter banks and Laplacian pyramid. It can be provided in the spatial domain as an efficient way for digital images that is completely specified by rectangular grids. In digital image curvelet transform decomposition poses numerous problems in practical applications:

* The approximated images have used overlapping windows, which increases the redundancy or blocking the effects due to its block-based transform properties.

* Ridgelet transform (for discrete images on rectangular coordinates) makes the implementation of the curvelet transform very challenging because it is defined on polar coordinate.

A double filter can achieve images by curvelet-like decomposition using multiscale decomposition for edge capturing. For instance, to tie link point discontinuities to linear structures one can use detection and then directional decomposition. Moreover, the relation of the curve scaling can be ensured by using an appropriate coordination between the directional basis and the scale and this is, actually, is linked to the number of directions [8]. Regarding the directional decomposition, Bamberger and Smith consider filter bank of images as an effective approach that was proposed by them. This method is featured by an important feature, which is critically sampled while getting optimal reconstruction. To obtain sparse representations of images, they propose a mixture of directional filter bank with a multi-resolution pyramid the resulted approach is named " pyramidal directional filter bank " [9].

\subsection{Pyramidal Directional Filter Banks}

The directional filter bank is designed to capture the high frequency components of images. Therefore, low frequency components are handled poorly. Actually, with the 
frequency partition shown in Figure 1, low frequencies would leak into several directional sub-bands, hence, directional filter bank does not provide a sparse representation for images and for situation improvement, low frequencies should be removed before to the directional filter bank. This provides another reason to the combined directional filter bank with a multi-resolution scheme [8].

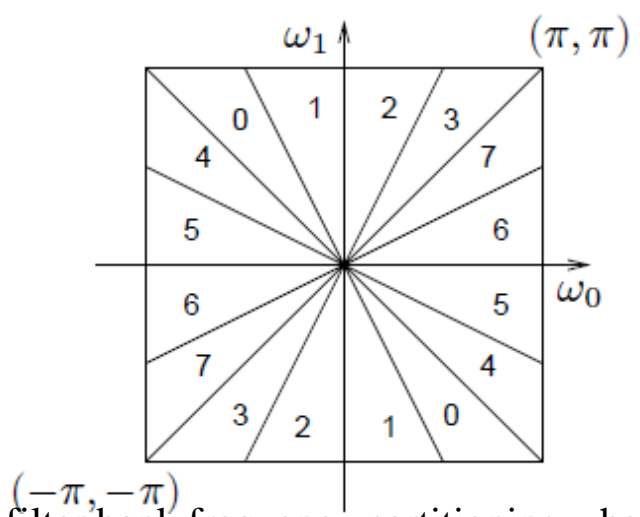

Figure 1: Directional filter bank frequency partitioning where $1=3$ and there are $2^{3}=8$ real wedge-shaped frequency bands [8]

One of the approaches used for multi-scale decomposition is Laplacian pyramid. Adelson and Burt firstly propose it. It generates a sample low-pass decomposition within every step. Laplacian pyramid applies sub-band decomposition on its band-pass images, which can be fed into a directional filter bank. This allows to capture the directional information (see Figure 2) [9]. The structure allows for different numbers of directions at each scale. Figure 2 (a): depicts the standard multi-scale decomposition in octave bands. In the case, the low-pass is subsampled and the high-pass not. Then, the directional filter bank applies directional decomposition for each path in the high-pass. Figure 2 (b): portrays the resulted frequency division. Here, the number of directions is increased with increasing the frequency [8].

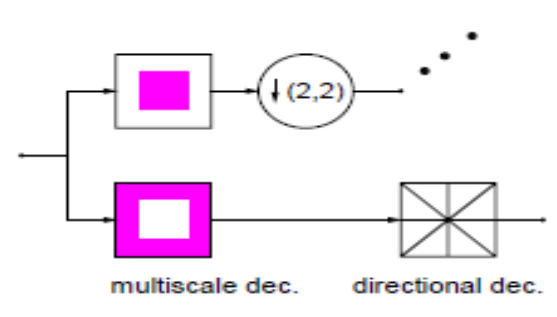

(a)

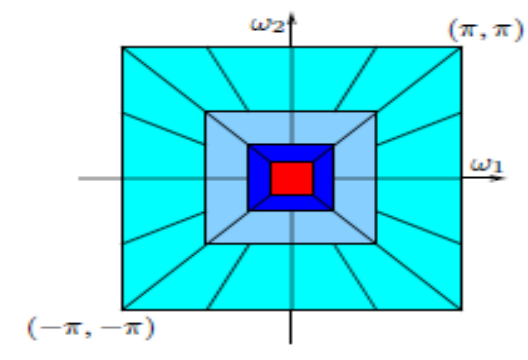

(b)

Figure 2: Pyramidal directional filter bank. (a) Block diagram,

(b) Resulting frequency division [9]

"The PDFB is a tight border when orthogonal filters are used in Laplacian pyramid and directional filter bank. The tight border is a very essential property in some applications". For instance, the error shown in the transform domain is similar in the reconstructed signal [9].

\section{Proposed Algorithm:}

At the beginning, image decomposition of Curvelet transform based in PDFB first and has the following steps [9]:

Step 1: Decomposing the object into a sequence of sub-bands. 
Step2: Center-frequency can be utilized for windowing the sub-bands into blocks of suitable size.

Step 3: For those blocks, the ridgelet transform is applied.

Our motivation in this work is that by smooth windowing, segments of smooth curves will seem straight in sub images. Therefore, ridgelet transform can be wellcaptured them. Sub-band decomposition can be used to control the number of ridgelets at multiple scales based on the fact that given scale of ridgelet lives in a certain subband. The window's size and sub-band frequency are coordinated and that curvelets have support obeying the key anisotropy scaling relation for curves: width $=$ length ${ }^{2}$. This will show that a PDFB where the number of directions is double at every subband. Now, the other finer scale in the pyramid (that is, applying a DFB with $\left(\mathrm{n}_{0}-[\mathrm{b} / \mathrm{j}]\right)$ levels to the bandpass image $b_{j}$ of the LP) satisfies those key properties of curvelets. Thus PDFB provides a filter bank approach for curvelet-like decomposition [9].

In the proposed algorithm the main goal is to study the effects of variation in size and cut-off frequencies of the low-pass filter over the curvelet coefficient especially when manipulate with aerial images. The decomposition operation is applied on the original image using curvelet transform with various scales, the number of coefficient, which obtained from curvelet transform depends on how many scales applied in this operation. The original image, which shown in Figure 3, is an aerial image for a place in Mosul, the image obtained from Remote Sensing center in Mosul university and the proposed algorithm will be applied on it with various filter sizes using two proposed approaches:

1. The main idea for the first approach is to change the filter size, which is used in curvelet operation related to image dimensions, therefore, a scalar factor overcomes to arrange the new filter size.

2. The second approach obtained the filter size dynamically by changing the parameters in curvelet transform.

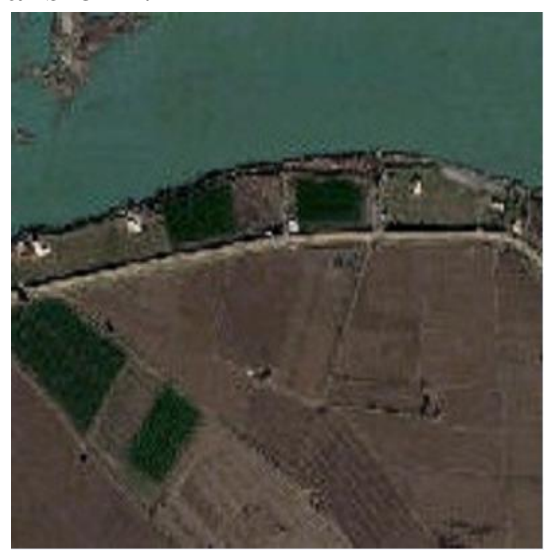

Figure 3: The original aerial image

A detailed explanation for the above two approaches can be seen in the following applied example:

\section{The First Proposed Approach}

In this approach, three experiments were implemented over the original image, therefore, various filter size are obtained as explained here.

The first experiment was implemented over the original image shown in Figure 3 with dimensions of $(400 * 400)$. Two scales are used to decompose the image using curvelet transform and nine coefficients can be obtained from curvelet. 
One of these coefficients must be selected to know the effects of varying the filter on it; coefficient two was selected in our experiment with dimensions of $(351 * 801)$ as shown in Figure 4.

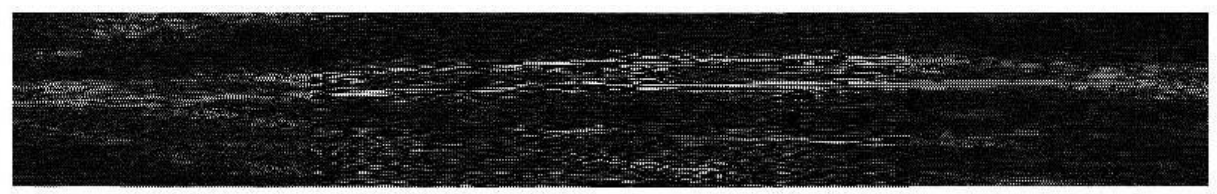

Figure 4: Coefficient 2 from Level 2

The histogram for this coefficient was introduced in Figure 5, the peak value at 137 as notice from the histogram can be used as threshold value.

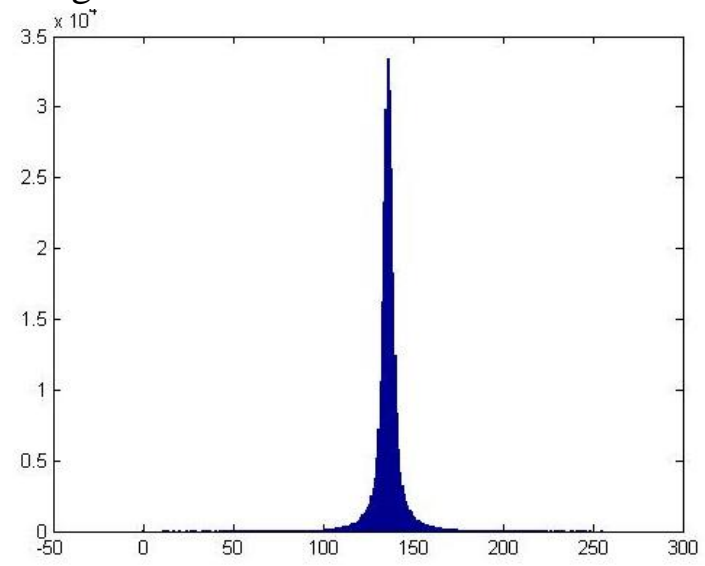

Figure 5: The Histogram for Coefficient 2

To make the proposed work more flexible, a value outside the window of $[+10$, 10] centered at the threshold consider being zero. This will make the less important pixels as black in the original image and edges appears in the resulted image as shown in Figure 6 that represents the effect of this filter over the original image.

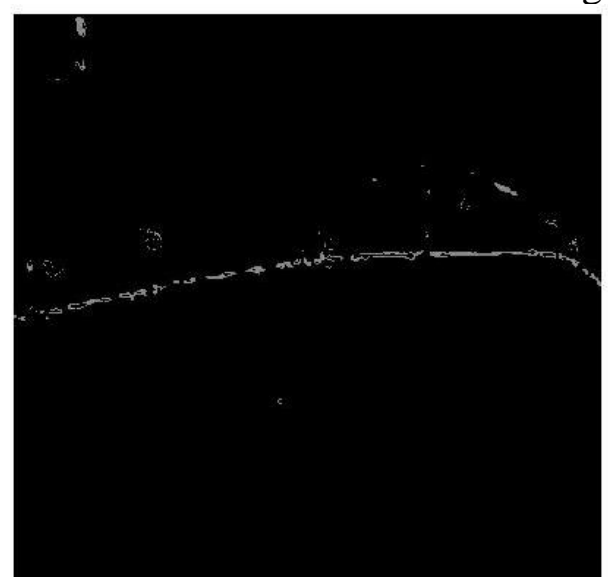

Figure 6: Resulted Image

As a result, after studying the effect of the filter on the original image, the obtained information was the filter dimension of $(800 * 800)$ can be build with frequency band of $(200,400,200)$. Table 1 shows the differents selection for filter size and filter dimension. After studying the above three experiments, as a result, an equation was developed for any dimensions in digital image $\left(\mathrm{N}^{*} \mathrm{~N}\right)$ when try to work with it under any scalar $(\mathrm{k})$. The filter size will be $(\mathrm{n} / \mathrm{k} * 2, \mathrm{n} / \mathrm{k} * 2)$ and its sub size will determined by the proposed equation:

$$
(\mathrm{n} / \mathrm{k}, 4 * \mathrm{n} / \mathrm{k}, \mathrm{n} / \mathrm{k}) \text {. }
$$




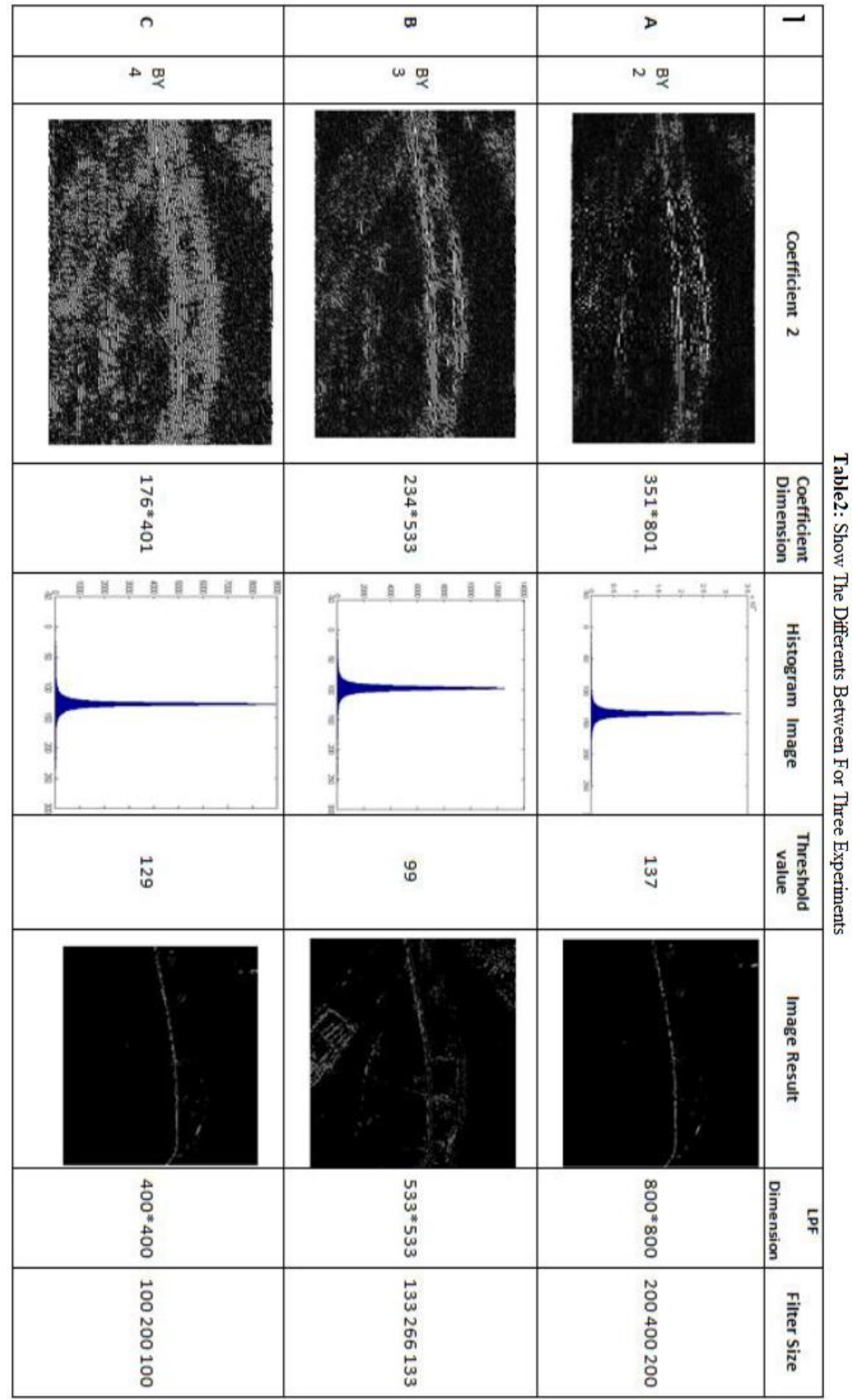




\section{The Second Proposed Approach}

As seen in first approach, decomposition operation using curvelet transform does not have flexibility to control filter size. Therefore, second approach will used to obtain filter size as needed as shown in three desired filter size experiments.

\section{A- Filter Size $(25,350,25)$}

After implementing curvelet transform on original image shown above in Figure $3,4,5$ with dimensions of $(400 * 400)$, it is not possible to obtain filter size $(25,350,25)$. To this end, many programming changes on curvelet program are made to create a LPF with desired size. When curvelet transform was applied using two scales, nine coefficients was obtained and coefficient two (shown in Figure 7) was selected to work on with dimensions of $\left(45^{*} 101\right)$.

Limitation of the filter dimension (i.e., 400*400) need a solution by modiffing on curvelet LPF filters. Nine coefficients were obtained when appling curvelet and the second coefficient was selected as shown in Figure 7 to be applied with $\left(45^{*} 101\right)$

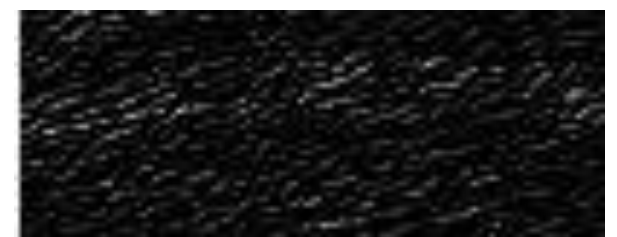

Figure 7: Coefficient 2 from Level 2

another modification must be applied on curvelet transform in order to create a filter with size $(25,350,25)$ :

- Image dimension must be divided by 16 .

- Implement the proper changes over low pass filter in order to get the desired filter size. Figure 8, show the high existing grary value (threshold value) which semes to be 122 in this experiment.

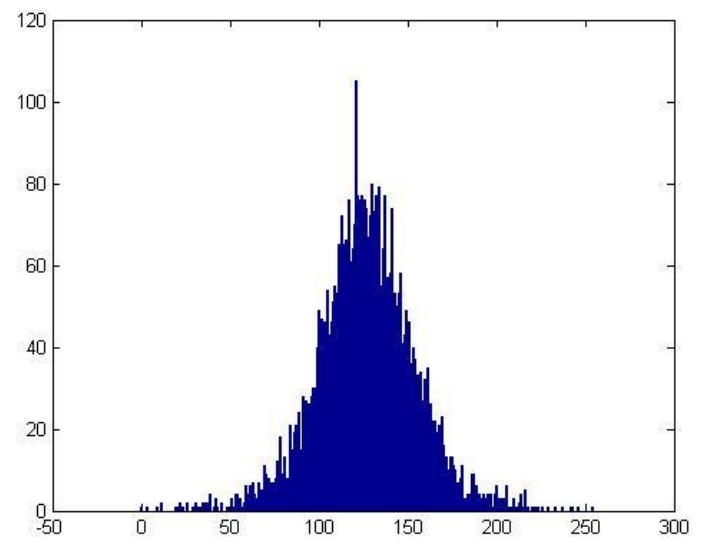

Figure 8: Histogram for cofficient 2 , filter with size $(25,350,25)$

Figure 9, shows the sub size of the desired filter which is $(25,350,25)$ and the Figure 10, shows the all low pass filter with the new size. 


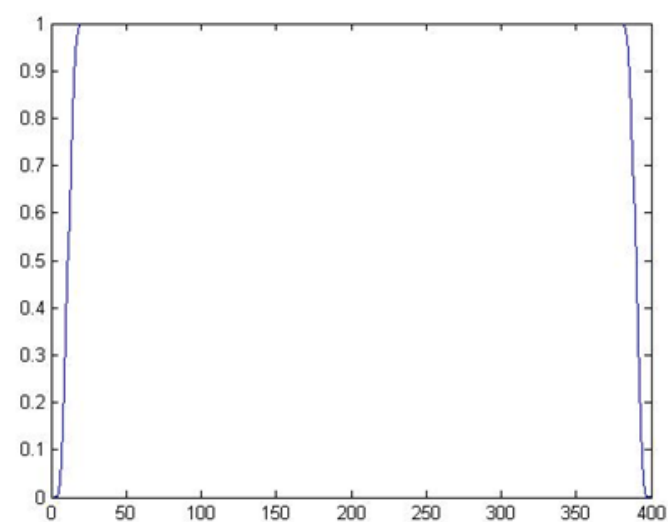

Figure 9: Low pass filter1

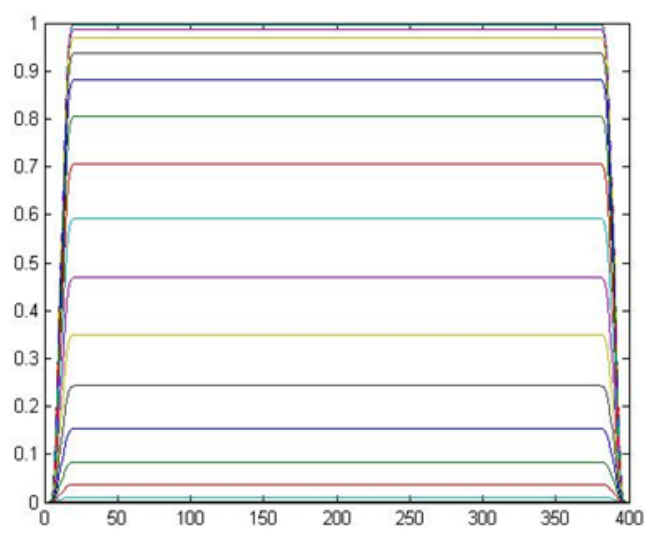

Figure 10: Low Pass Filter1*Lowpass filter2

Finally, expand the threshold value by $[+10,-10]$ and replacing all uases ouer or less than threshould value by zero will extract the edges as shown in Figure 11.

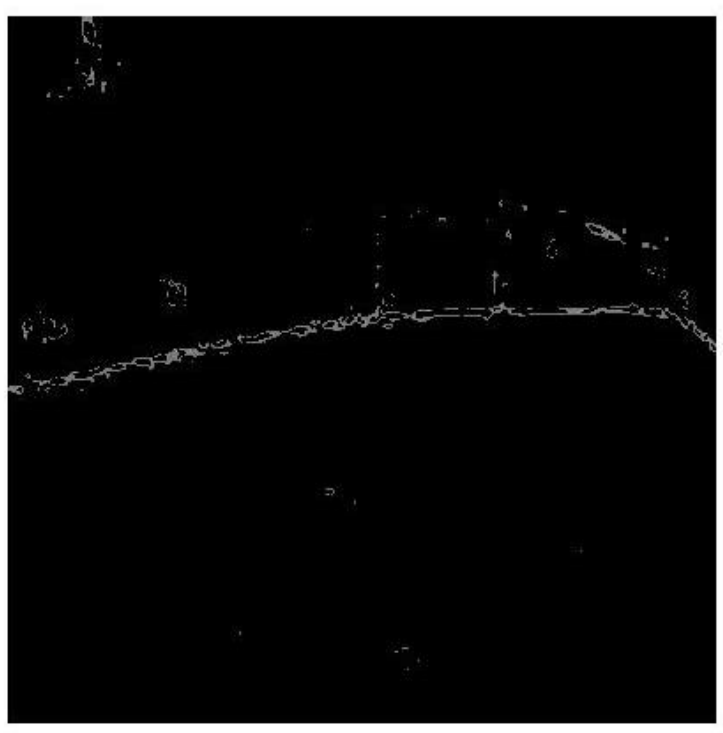

Figure 11: Resulted Image

After studying Table 2 with three experiments, an important notice is raised, the filter size does not affect the dimensions of the coefficient, it affects the details information in the coefficient. 


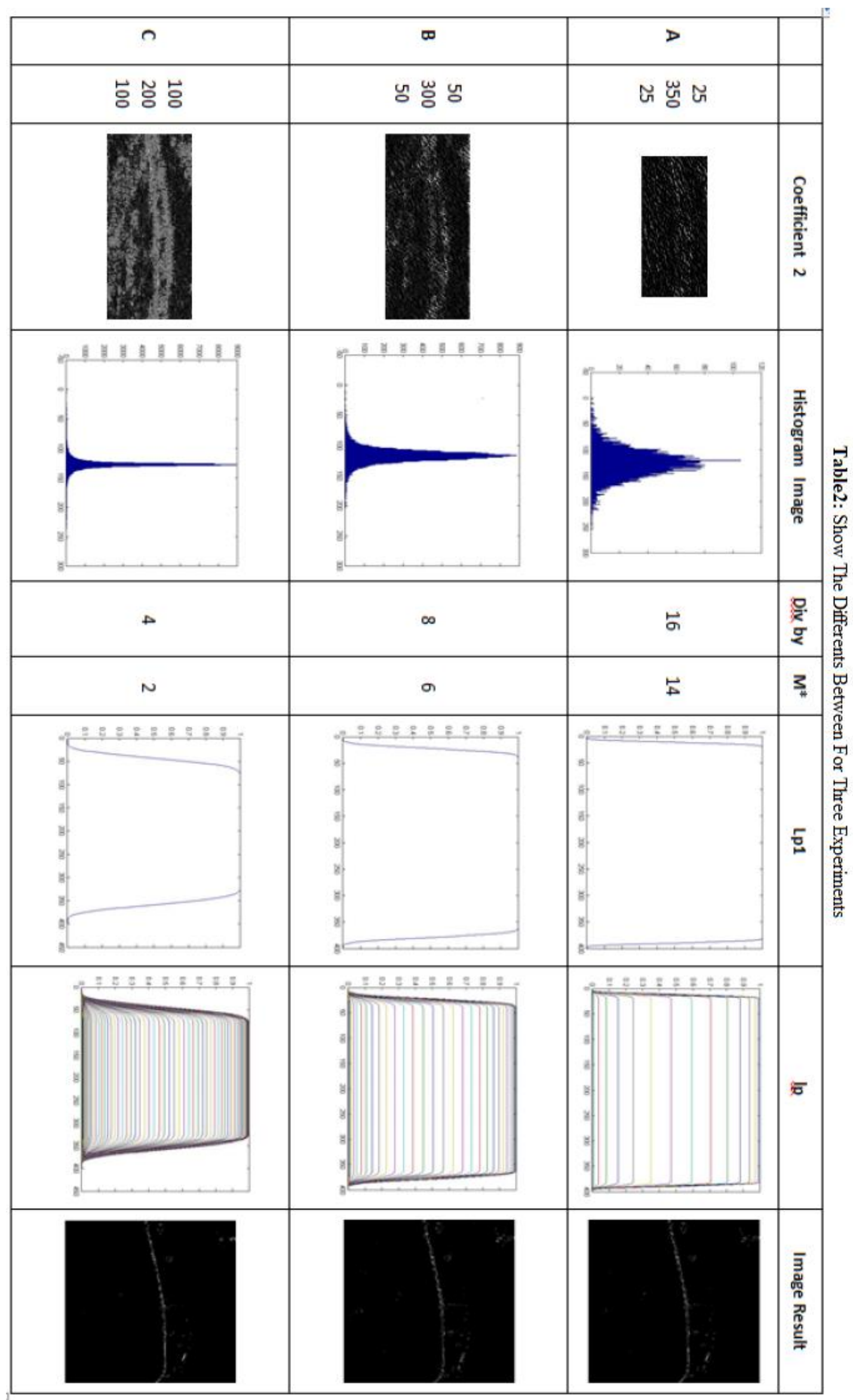




\section{Conclusion And Future Work}

From the result of applied example, this research shows that a deep effect of decomposing the aerial images by curvelet. Moreover, the practical side extracts the benefits of using $\mathrm{N}$-cut algorithm merged with curvelet transformation in addition to following:

- Segmentation based on choosing a threshold value gives appositive effect to get ROI from the segmented parts.

- AutoDetect of threshold value gives optimal segmentation.

- One of the important points given by this research is the main effect of the cut-off frequency on the segmentation through its effect on the image parameters.

- A designed filter for curvelet decomposition by selecting a proper low/high cut-off frequency to fix the band pass region, our approach gives better segmentation than the other ones. 


\section{REFERENCES}

[1] A. Abdullah, M. Ali, I. Al_Barazanchi, 2019, "Improving face recognition by elman neural network using curvelet transform and HSI color space", Periodicals of Engineering and Natural Sciences, Vol.7, No.2, June 2019, pp.430-437, ISSN 2303-4521.

[2] B. Asim, H. Cresswell, 2014, “ Curvelet Transform To Study Scale-Dependent Anisotropic Soil Spatial Variation”, Elsevier Geoderma 213,PP 589-599.

[3] Djimeli A. , Tchiotsop D., R. Tchinda, 2013, "Analysis Of Interest Points Of Curvelet Coefficients Contributions Of Microscopic Images And Improvement Of Edges", Signal \& Image Processing: An International Journal (Sipij) Vol.4, No.2.

[4] J. Ishrat, 2008, " Image Retrieval Using Discrete Curvelet Transform", Monash University, Australia, November.

[5] K. kishore, May - June, 2013, "medical image fusion using curve let transform", international journal of electronics and communication engineering \& technology (ijecet), Volume 4, Issue 3, pp. 193-201.

[6] L. Ying, L. Demanet and E. Cand`es, "3D Discrete Curvelet Transform", Applied and Computational Mathematics, MC 217-50, Caltech, Pasadena, CA.

[7] M. Oraiqat, F. Awwad, 2004, "A Comparison Of Image Enhancement Using Curvelet Transform With Multiscale Gradient And Retinex Operators", Wseastrans On Eledornics, Issue3 Vol.

[8] N. Minh DO, 2001, "Directional Multiresolution Image Representations", Bachelor of Engineering In Computer Engineering, University Of Canberra, Australia.

[9] N. Minh Doy And M. Vetterliy, 2001, "Pyramidal Directional Filter Banks And Curvelets", IEEE International Conference On Image Processing (ICIP), Thessaloniki, Greece.

[10] P. Tirveedi, CH. Pullarao, 2014, “ An Efficient Cum Improved Resolution Enhancement Using Zero Padding And Curvelet Transforms", International Journal Of Engineering Trends And Technology (IJETT) - Vol.15 Number 1.

[11] Padmanjali, DC. Shubhangi, 2018, "Brain Tumor MR Image Fusion Using Most Dominant Features Extraction From Wavelet And Curvelet Transforms", International Research Journal Of Engineering And Technology (IRJET), Volume: 05 Issue: 05, ISSN: 2395-0056.

[12] Raju*, T. Sreenivasulu Reddy and M. Sivasubramanyam, 2016, "Denoising of Remotely Sensed Images via Curvelet Transform and its Relative Assessment", Sri Venkateswara University College of Engineering, S.V. University, Tirupati, A.P., India, Procedia Computer Science 89, 771 - 777.

[13] Ren , J. Yang., 2012, "A Novel Color Microscope Image Enhancement Method Based On HSV Color Space And Curvelet Transform", IJCSI International Journal Of Computer Science Issues, Vol. 9, Issue 6, No 2, ISSN: 1694-0814.

[14] S.Hejazi, M. Shahbazian, et al..., 2013, " Angle based curvelet transform method for ground roll removal ", Australian Journal of Basic and Applied Sciences, 7(4): 123-132, ISSN 1991-8178.

[15] T. Ahmad, Li X., 2012, "An Efficient Curvelet Based Miso Image Super Resolution Algorithm Using Kurtosis As Input", Journal Of Computational Information Systems 8:22, 9415-9423. 